\title{
MONODISPERSE MICROPARTICLES OBTAINED USING SPG MEMBRANE TECHNIQUE
}

\author{
F. R. Steinmacher ${ }^{1}$, A. Musyanovych ${ }^{2}$, K. Landfester ${ }^{2}$, C. Sayer ${ }^{1}$, P. H. H. Araujo ${ }^{1}$ \\ ${ }^{1}$ Federal University of Santa Catarina, Chemical Engineering Department, Florianópolis, Brazil \\ ${ }^{2}$ Max Planck Institute for Polymer Research - Mainz, Germany \\ E-mail: Pedro@enq.ufsc.br
}

\begin{abstract}
Biodegradable particles with narrow size distribution are desirable as drug delivery systems. Monodisperse microparticles (MPs) prepared with degradable polymer for application as drug delivery system was designed. PLLA microparticles (MPs) with size around 2 and $5 \mu \mathrm{m}$ were prepared using the SPG membrane emulsification technique. The ratio value between mean particle size and mean pore size may only kept constant once the operating conditions are optimized. Hydrophobic dye as N-(2,6diisopropylphenyl)perylene-3,4-dicarbonacidimide (PMI) was encapsulated. The morphology was evaluated using SEM and fluorescence microscopy. PMI - labeledmicroparticles represent their ability of encapsulate hydrophobic drugs. The polymer chosen exhibited enzymatic degradation. These initial results demonstrate the potencial for application as drug delivery system that requires enzyme-triggered release.
\end{abstract}

\section{INTRODUCTION}

Biodegradable particles with narrow size distribution are desirable as drug delivery systems, since several reports have shown that cellular uptake is correlated with particle size (GAUMET et al., 2007).

Drug-aerosol inhalation is one of the best pathway to lung diseases treatment, and the major advantages of inhalation therapy are the non-invasive nature and the drug is driven directly to the target(BYRON, 1990). Beyond, administration via oral inhalation is receiving considerable attention also because the lung has a huge surface area for adsorption. According to Byron (1990), even compounds with very small absorption rates can be transferred to the systematic circulation, avoiding many of the first pass metabolism problems.

Successful delivery of inhaled particles is governed by their deposition patterns, which are mainly controlled by particle size and density (KLEINSTREUER et al., 2008). For deep lung deposition, it is required microparticles size between 1 and $5 \mu \mathrm{m}$ (HUREAUX et al., 2009). Besides microparticles allow adequate delivery to lungs, micro- and nano-particles can provide controlled release of drugs and targeting at cellular levels.

It is already established in literature that the microporous membrane technique produces monodisperse droplets that can be further polymerized or solidified, resulting in a very narrow particle size distribution (ITO and MAKINO, 2004). This work proposes to prepare narrow size 


\section{9 a 22 de outubro de 2014 \\ Florianópolis/SC}

PLLA particles distribution with a facile approach and only one-step emulsification for application as drug delivery polymeric system of hydrophobic compounds to the lungs.

\section{EXPERIMENTAL}

\subsection{Materials}

Poly-L-Lactide Acid (PLLA, Biomer Biopolyesters, Biomer L9000®, number average molecular weight, $M n \approx 66,500 \mathrm{~g} / \mathrm{mol}$, weight average molecular weight $M w \approx 145,000 \mathrm{~g} / \mathrm{mol}$, determined by gel permeation chromatography (GPC) in chloroform) and chloroform (Arcos Organics), chosen as solvent, were used to prepare biodegradable microparticles. Sodium dodecyl sulfate (SDS, Merck) was used as anionic surfactant and distilled water was used as continuous phase. All chemicals were used without further purification. The fluorescent perylene dye N-(2,6diisopropylphenyl)perylene-3,4-dicarbonacidimide (PMI, $\lambda_{\mathrm{exc}} / \lambda_{\mathrm{em}}: 488 / 520 \mathrm{~nm}$ ) was encapsulated into the PLLA microparticles. For enzymatic degradation, proteinase K (lyophilized

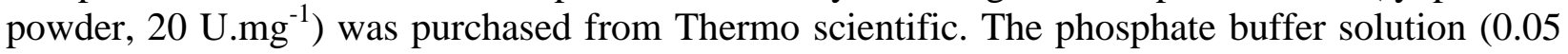
$\mathrm{M}, \mathrm{pH}$ 7.4) was freshly prepared from monobasic and dibasic sodium phosphate (Vetec).

\subsection{Preparation of PLLA Microparticles}

PLLA was chosen as the model polymer to prepare the microparticles due to its biodegradability. The PLLA microparticles were prepared by an emulsion solvent evaporation technique using two different processes of emulsification. The first one consists of a homogenizer (ultraturrax) at a stirring rate of 18,000 rpm for $4 \mathrm{~min}$. The second approach makes uses of a Shirasu Porous Glass (SPG) module, according to formulations shown in Table 1. This technique is based on pressing the dispersed phase through a membrane with uniform and specific pore size into the continuous phase. For that, hydrophilic SPG membranes tube-shaped (10 mm outer diameter and thickness of $0.7 \mathrm{~mm}$ ) were provided by SPG Technology Co. Ltd. (Sadowara, Japan). The membranes were regenerated after use by dipping in chloroform, dimethyl sulfoxide and then, distilled water for 15 minutes in each solvent using an ultrasonication bath. The dispersed phase, composed of chloroform (2.5 g) and PLLA, was stored in a pressure-tight vessel that was connected to a nitrogen gas inlet attached to a pressure gauge PG-30-102R-N-ND (COPAL Eletronics, Japan). By appling an adequate pressure, the dispersed phase passed through the membrane into the continuous phase, composed of a SDS aqueous solution, which was kept under stirring during the emulsification in order to detach the formed droplets from the pores at the interface between membrane and continuous phase, and to prevent the creaming of the droplets. The next step was the solvent evaporation, that was carried out at $40{ }^{\circ} \mathrm{C}$ overnight using a magnetic stirrer. To obtain monodisperse microparticles, membranes with a mean pore size of 2 and $5 \mu \mathrm{m}$ were used and driving pressures of 60 and $10 \mathrm{kPa}$ were applied, respectively. When nanocapsules were added to the dispersed phase, the free surfactant was removed by centrifugation for $50 \mathrm{~min}$ at $5,600 \mathrm{rpm}$ and the nanocapsules were redispersed in chloroform using an ultrasonication bath. Dye-loaded microparticles were prepared replacing the chloroform that composes the dispersed phase by a $0.02 \mathrm{wt} \%$ PMI solution. 
Table 1 - Formulation of microparticles obtained by SPG membrane technique.

\begin{tabular}{lccccc}
\hline Reactants & UT 4- $1^{\mathrm{a}}$ & MT 2-1 $^{\mathrm{b}}$ & MT 5- $^{\mathrm{c}}$ & MT 5-4 & MT 5-1 ${ }^{\mathrm{c}}$ \\
\hline PLLA (g) & 0.040 & 0.080 & 0.080 & 0.040 & 0.040 \\
Chloroform (g) & 2.500 & 2.500 & 2.500 & 2.500 & 2.500 \\
SDS (g) & 0.030 & 0.018 & 0.018 & 0.018 & 0.030 \\
Water (g) & 12.00 & 12.00 & 12.00 & 12.00 & 12.00 \\
\hline Emulsification Step & & & & \\
\hline Driving Pressure (kPa) & - & 60 & 10 & 10 & 10 \\
Stirring Speed (rpm) & 18,000 & 500 & 750 & 750 & 750 \\
\hline Solvent Evaporation & & & & & \\
\hline Temperature $\left({ }^{\circ} \mathrm{C}\right)^{\mathrm{c}}$ & 40 & 40 & 40 & 40 & 40 \\
Stirring Speed (rpm) & 750 & 500 & 750 & 750 & 750 \\
\hline
\end{tabular}

a)Emulsification by ultraturrax for 4 min at 18,000 rpm; b)Emulsification by membrane technique using a 2 um pore size hydrophilic membrane at room temperature; c) Emulsification by membrane technique using a 5 m pore size hydrophilic membrane at room temperature.

\subsection{Characterization}

Particle Size and Morphology: The morphology of the nanocapsules was evaluated by scanning electron microscopy (SEM) using a field emission microscope (LEO (Zeiss) 1530 Gemini, Oberkochen, Germany) at an accelerating voltage of $0.5 \mathrm{kV}$. The samples were prepared by diluting the microparticles in distilled water, then one droplet of the sample was placed onto silica wafers and dried under ambient conditions. Microparticle size was analyzed using image analysis software Sizemeter. Average particle diameter, standard deviation and particle size distribution were determined from data sets of at least 300 particles.

Degradation of MPs: For degradation studies, proteinase $\mathrm{K}\left(10 \mathrm{mg}^{\mathrm{m}} \mathrm{ml}^{-1}\right)$ was dissolved in $0.05 \mathrm{M}$ $\mathrm{PBS}$ at $\mathrm{pH}$ 7.4. Incubation of $15 \mathrm{mg}$ of MPs with the enzyme was achieved under gentle shaking conditions at $37{ }^{\circ} \mathrm{C}$. MPs were previously purified by repetitive centrifugation for $20 \mathrm{~min}$ at 3,000 rpm (MiniSpin Eppendorf) and redispersed in order to remove SDS. The morphologies of PMI-loaded MPs degraded by proteinase $\mathrm{K}$ were verified using an epifluorescence microscope Olympus BX41 with filter set (blue,U-MWU2, $\lambda_{\text {exc }} / \lambda_{\text {em }}: 330-385 / 420 \mathrm{~nm}$, green, U-MWB2, $\lambda_{\text {exc }} / \lambda_{\text {em }}: 460$ - $490 / 520 \mathrm{~nm}$ and violet, U-MGW2, $\lambda_{\text {exc }} / \lambda_{\text {em }}: 510$ - $550 / 590 \mathrm{~nm}$ ) equipped with a 3.3 mpixel camera (QImaging, Surrey, BC, Canada). All images were acquired using QCapture Pro 5.1 software (QImaging) without post-acquisition processing. 


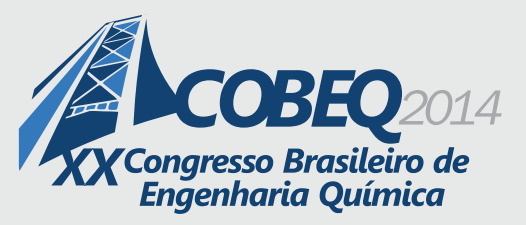

19 a 22 de outubro de 2014
Florianópolis/SC

\section{RESULTS AND DISCUSSION}

Several works in literature prepared microparticles using a high-speed homogenizer or ultrasonication as emulsification steps. Although, some attempts were carried out applying ultrasonication technique, the particles always presented sizes in nanoscale. Meanwhile, highspeed homogenizer at 18,000 rpm for $4 \mathrm{~min}$ resulted a broad microparticles size distribution. A comparative study between SEM images of PLLA microparticles obtained using different emulsifications technique is shown in Figure 1. SEM images (Figure 1a) confirmed that the size distribution is very broad, due to the fact that the size of the particles prepared by this method is difficult to be controlled.

In order to obtain monomodal particles size distribuition, SPG-membrane technique was investigated. The effect of the mean pore size of the membrane on the average microparticles diameter and size distribution was evaluated. For that, membranes with mean pore sizes of 2 and $5 \mu \mathrm{m}$ were investigated.

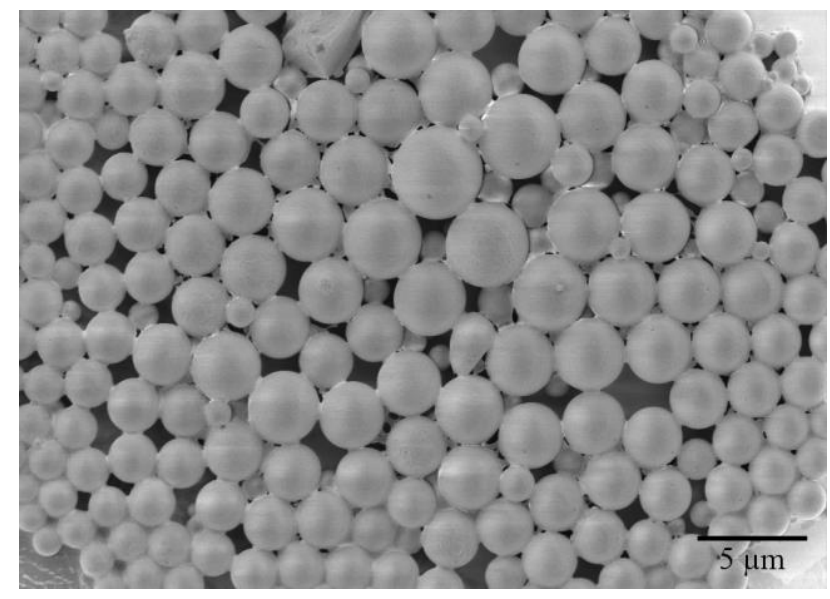

(a)

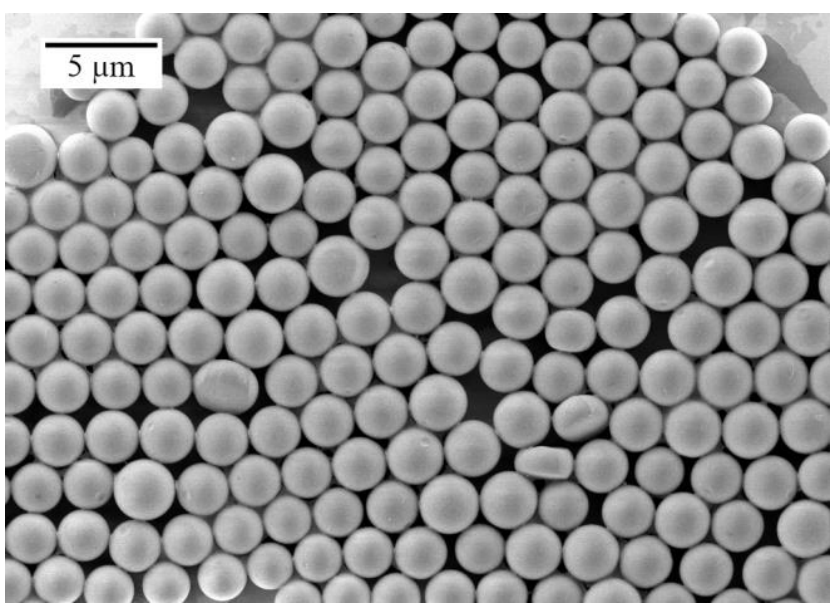

(b)

Figure 1 - Comparison between SEM images of PLLA microparticles obtained using (a) an ultraturrax at $18000 \mathrm{rpm}$ for 4 min (UT 4-1) and (b) SPG membrane technique with 2 um pore size membrane (MT 2-1).

Besides of homogeneity of the pores, the driving pressure is also a parameter that affects the size distribution of the microparticles. For instance, driving pressure and stirring speed during the emulsification step were adapted for which pore size membrane, as shown in Table 1 . The driving pressure was adjusted to $60 \mathrm{kPa}$ and $10 \mathrm{kPa}$ for the 2 and $5 \mu \mathrm{m}$ pore size membranes, respectively (these values were chosen based on preliminary tests). From a comparison between MT 2-1 and MT 5-1, it can be observed that the mean particle size increased from 2.30 to 6.23 $\mu \mathrm{m}$ with increasing the mean pore size of SPG membrane from 2 to $5 \mu \mathrm{m}$, respectively. The size 


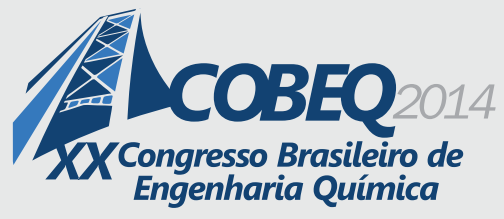

19 a 22 de outubro de 2014

Florianópolis/SC

of particles is quite similar to the pore size of the membrane. Basically, these results are in agreement with the results obtained by Vladisavljevic et al. (2006). The authors varied the mean pore size of membrane from 5.4 to $20.3 \mu \mathrm{m}$ and observed that, after direct emulsification, the ratio between mean particle size and mean pore size was constant and independent on the mean pore size. The ratio value between mean particle size and mean pore size may only kept constant once the operating conditions are optimized.

Bigger is the pore size membrane, generally lower is driving pressure required and higher is the stirring speed to detach the microdroplets formed at the interface between SPG membrane and continuous phase. To ensure the regular detachment of the droplets from the pore outlets, shear stress was applied at the membrane-continuous phase interface using a magnetic stirrer. It was already reported that in membrane emulsification the stirring speed of the continuous phase has a significant effect on the size and size distribution of the droplets ( $\mathrm{OH}$ et al., 2011). It was observed that, when $5 \mu \mathrm{m}$ pore size membrane was used, the stirring speed was a critical parameter to obtain stable and monodisperse particles. While for the $2 \mu \mathrm{m}$ pore size membrane, the stirring speed was $500 \mathrm{rpm}$, for the $5 \mu \mathrm{m}$ one, it was necessary to increase to $750 \mathrm{rpm}$. This fact might also be due to the increase of inertia force that is related with big droplets. According to Nazir et al. (2010) in cross-flow emulsification, the forces acting on the forming droplet are mainly the interfacial tension force (that keeps the droplet connected to the pore) and the shear force (due the continuous phase flow that tries to remove the droplet). However, in case of big droplets (>10_m), also the buoyant and inertia forces need to be considered.

The PLLA concentration in the disperse phase also plays a role in membrane emulsification. Spherical-shaped microspheres (MT 5-1) with a smooth surface were obtained, as can be observed in Figure 2a. Regarding to the amount of PLLA, it was varied from 0.04 to 0.08 g, MT 5-4 and MT 5-1, respectively. It was observed that the mean particle diameter increased from 4.93 to $6.23 \mu \mathrm{m}$, when the amount of PLLA was enhanced from 0.04 (MT 5-4) to $0.08 \mathrm{~g}$ (MT 5-1). Figure 2b shows the effect of PLLA amount on the particle size distribution.

In contrast to our results, Ma et al. (1999) observed that small increments in PLA concentration decreased the particle diameter and attributed that to the increase of viscosity of dispersed phase. The authors studied the effect of PLA concentration on the particle diameter using a SPG membrane with a mean pore size of $5.2 \mu \mathrm{m}$, dichloromethane as dispersed phase and lauryl alcohol as co-surfactant. The decreased in particle size was attributed by the authors to the greater disperse phase viscosity. When the viscosity of the oil phase increases, the flow of this phase inside the pores of the membrane becomes slower, consequently, the droplet size becomes smaller. However, the rise of particle size with the increase of PLLA concentration might be attributed to the solvent evaporation process. Although greater viscosity of disperse phase could decrease the droplet size, during the solvent evaporation process, the particles became bigger due to the higher polymer concentration in each droplet. This fact might explain the bigger diameter obtained after solvent evaporation of MT 5-1. 


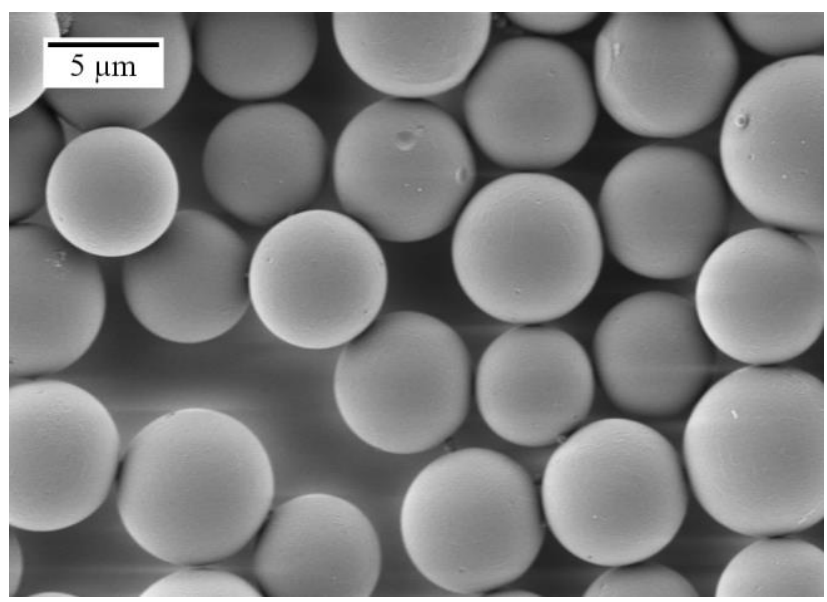

(a)

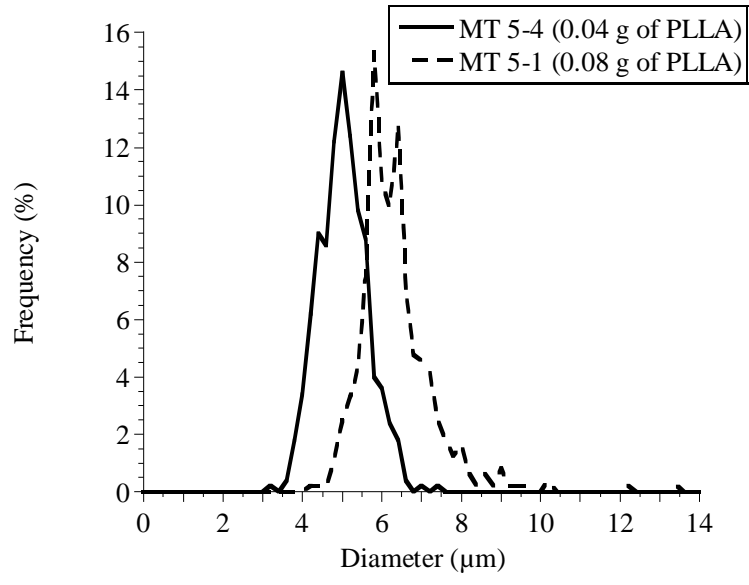

(b)

Figure 2 - (a) SEM image of PLLA microparticles MT 5-1 microparticles obtained by membrane technique using $5 \mu \mathrm{m}$ pore size of membrane. (b) Effect of PLLA amount on particle diameter distribution.

During the droplet formation process, the surfactant molecules adsorb onto the newly formed oil-water interface and reduce the interfacial tension thus, facilitating droplet formation. The rate of transfer of the surfactant molecules from the bulk solution to the newly formed oilwater interface partly depends on the continuous-phase flow (OH et al., 2011). With an increase in the amount of SDS from $0.018 \mathrm{~g}$ to $0.030 \mathrm{~g}$, neither the mean particle sizes, $4.90 \mu \mathrm{m}$ and 4.87 $\mu \mathrm{m}$, respectively, nor the particle size distributions varied, as shown in Figure $3 \mathrm{a}$.

This fact indicates that the lower amount of surfactant used (0.018 g, MT 5-4) was enough to cover the whole particle surface to stabilize the droplet. Therefore, the droplet size was controlled by the emulsification conditions, for instance, pore size of membrane, driving pressure and stirring speed of the continuous-phase. Figure presents SEM images of MT 5-11 microparticles obtained with higher amount of surfactant in the continuous phase at $750 \mathrm{rpm}$ agitator speed during emulsification and solvent evaporation steps.

PMI - labeled-microparticles represent their ability of encapsulate hydrophobic drugs. After 3 days, no significant release of PMI was detected from the PLLA microparticles. This fact might be due to the hydrophobic character of dye, big particle size and also because of the hydrolysis degradation behavior. For PLLA microparticles, bulk degradation occurs by centrifugal hydrolysis, with faster degradation rate in the center than at the surface, due to an autocatalytic effect of acidic degradation products trapped inside the polymer matrix (PARK, 1995; ANDERSON and SHIVE., 2012). The release of hydrophobic drugs upon PLLA microparticles degradation might be considered. To demonstrate this, the degradation of PLLA microparticles by proteinase $\mathrm{K}$ were monitored via fluorescence microscopy (Figure ). 


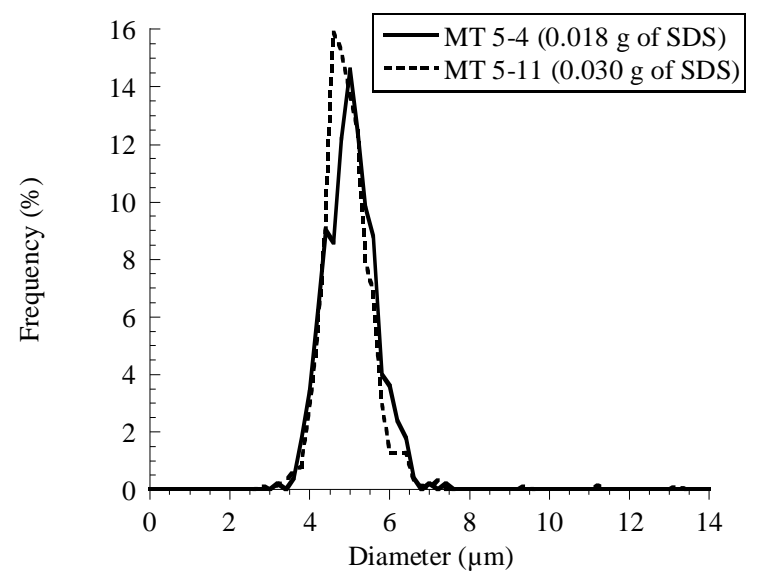

(a)

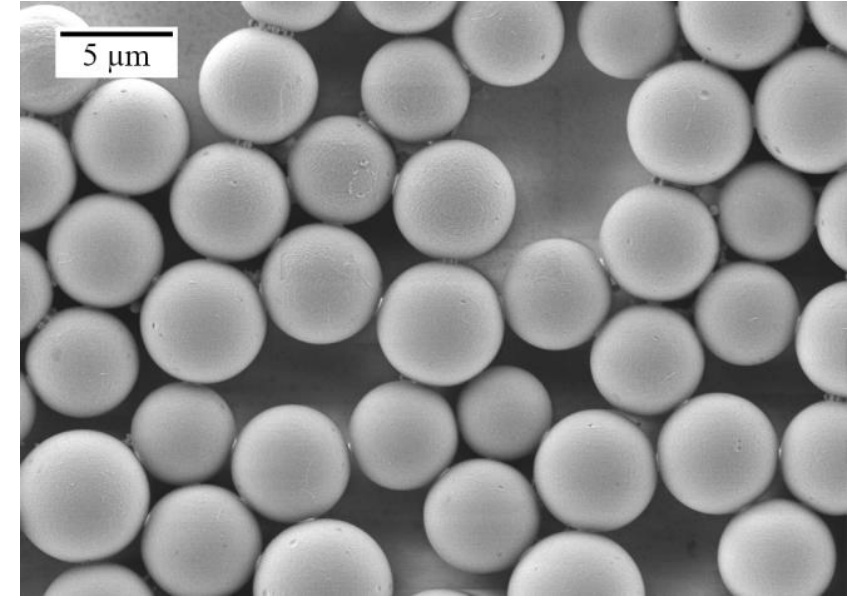

(b)

Figure 3 - (a) Effect of surfactant concentration in continuous phase on particle diameter distribution. (b) SEM image of MT 5-11 PLLA microparticles.

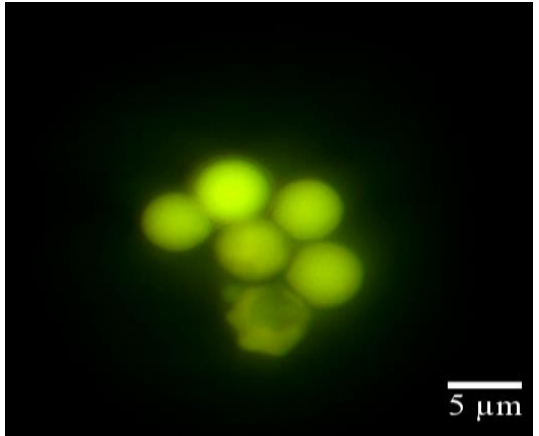

(a)

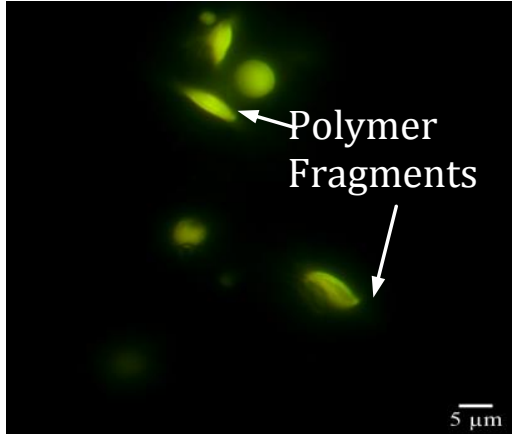

(b)

Figure 4 - Enzymatic degradation of multicompartment particles MT 5-8 incubated with proteinase K. Fluorescence microscopy images (a) before treatment and after 1 day (b) of PMIlabeled particles.

\section{CONCLUSIONS}

Monodispersed PLLA microparticles were prepared using the SPG membrane emulsification technique. It was observed that the average particle size decreased, decreasing the pore diameter of SPG membrane. The ratio value between mean particle size and mean pore size may only kept constant once the operating conditions are optimized. SEM and fluorescence microscopy were essential for the characterization of multicompartment particles. PMI - labeled-microparticles represent their ability of encapsulate hydrophobic drugs. The polymer chosen exhibits enzymatic degradation. These initial results demonstrate the potencial for application as drug delivery system that requires enzyme-triggered release. 


\section{REFERENCES}

ANDERSON, J. M. and SHIVE, M. S. Biodegradation and biocompatibility of PLA and PLGA microspheres. Adv. Drug Deliv. Rev. v. 64, p. 72-82, 2012.

BYRON, P. Determinants of drug and polypeptide bioavailability from aerosols delivered to the lung. Advanced Drug Delivery Reviews, n. 5, p. 107 - 132, 1990.

GAUMET, M.; GURNY, R.; DELIE, F. Fluorescent biodegradable PLGA particles with narrow size distributions: preparation by means of selective centrifugation. International journal of pharmaceutics, $\mathrm{v}$. 342, n. 1-2, p. 222-30, 5 set. 2007.

HUREAUX, J. et al. Lipid nanocapsules: ready-to-use nanovectors for the aerosol delivery of paclitaxel. European journal of pharmaceutics and biopharmaceutics, v. 73, n. 2, p. 239-46, out. 2009.

ITO, F.; MAKINO, K. Preparation and properties of monodispersed rifampicin-loaded poly(lactide-coglycolide) microspheres. Colloids and surfaces. B, Biointerfaces, v. 39, n. 1-2, p. 17-21, 25 nov. 2004.

KLEINSTREUER, C.; ZHANG, Z.; DONOHUE, J. F. Targeted drug-aerosol delivery in the human respiratory system. Annual review of biomedical engineering, v. 10, p. 195-220, jan. 2008.

MA, G.; NAGAI, M.; OMI, S. Preparation of uniform poly(lactide) microspheres by employing the Shirasu Porous Glass (SPG) emulsification technique. Colloids and Surfaces A: Physicochemical and Engineering Aspects, v. 153, p. 383-394, 1999.

NAZIR, A.; SCHROËN, K.; BOOM, R. Premix emulsification: A review. Journal of Membrane Science, v. 362, n. 1-2, p. 1-11, out. 2010.

$\mathrm{OH}, \mathrm{D}$. H. et al. Effect of process parameters on nanoemulsion droplet size and distribution in SPG membrane emulsification. International journal of pharmaceutics, v. 404, n. 1-2, p. 191-7, 14 fev. 2011.

PARK, T. G. Degradation of poly ( lactic-co-glycolic acid ) microspheres : effect of copolymer composition. Biomaterials, v. 16, n. 15, p. 1123-1130, 1995.

VLADISAVLJEVIĆ, G. T.; SHIMIZU, M.; NAKASHIMA, T. Production of multiple emulsions for drug delivery systems by repeated SPG membrane homogenization: Influence of mean pore size, interfacial tension and continuous phase viscosity. Journal of Membrane Science, v. 284, n. 1-2, p. 373-383, 1 nov. 2006.

\section{ACKNOLOGMENT}

The authors thank the financial support from $\mathrm{CNPq}$ - Conselho Nacional de Desenvolvimento Científico and BMBF - Bundesministerium für Bildung und Forschung. 